\title{
Representation of optical fields using finite numbers of bits
}

\author{
Ayça Özçelikkale* and Haldun M. Ozaktas \\ Department of Electrical Engineering, Bilkent University, TR-06800, Ankara, Turkey \\ ${ }^{*}$ Corresponding author: ayca@ee.bilkent.edu.tr
}

Received February 10, 2012; revised March 20, 2012; accepted April 2, 2012; posted April 13, 2012 (Doc. ID 162828); published June 4, 2012

\begin{abstract}
We consider the problem of representation of a finite-energy optical field, with a finite number of bits. The optical field is represented with a finite number of uniformly spaced finite-accuracy samples (there is a finite number of amplitude levels that can be reliably distinguished for each sample). The total number of bits required to encode all samples constitutes the cost of the representation. We investigate the optimal number and spacing of these samples under a total cost budget. Our framework reveals the trade-off between the number, spacing, and accuracy of the samples. When we vary the cost budget, we obtain trade-off curves between the representation error and the cost budget. We also discuss the effect of degree of coherence of the field. (c) 2012 Optical Society of America

OCIS codes: $\quad 070.2025,070.0070,110.3055,030.0030,100.3020,350.5730$.
\end{abstract}

In this letter, we consider the problem of representation of a finite-energy nonstationary optical field, with a finite number of bits. The optical field $f(x)$ is to be represented with a finite number of finite-accuracy, equidistant samples. Our aim is to investigate the relationships between the goodness and the cost of this representation of the field, and the accuracies and locations of the samples. For instance, we would like to learn whether it is better to use a small number of samples with higher accuracies or a large number of samples with lower accuracies. (With high amplitude accuracy, we refer to a high number of amplitude levels that can be reliably distinguished.)

We introduce a cost budget, which limits how much we can simultaneously increase the number of samples and the accuracies of the samples. Under a given total budget, we determine the optimal number of samples and the optimal sampling interval. By varying the budget, we obtain trade-off curves between the representation error and the cost budget.

Such investigations will yield a better understanding of the information-theoretical relationships inherent in optical fields: how well the field values at particular points can represent the whole field has to do with how much information these values carry about the rest of the field. Many aspects of the information-theoretical relationships in optical fields have been studied (for instance, [1-10]). Our purpose is to contribute to this understanding by utilizing concepts from signal analysis and information theory.

Let $f(x)$ be a zero-mean proper complex Gaussian random field (random process). $M$ finite-accuracy equidistant samples of $f(x)$, with the sampling interval $\Delta_{x}$, will be used to provide a representation of $f(x)$. The limited amplitude resolution (finite accuracy) of the samples is modeled through an additive noise process

$$
s_{i}=f\left(\xi_{i}\right)+m_{i}
$$

where $x=\xi_{1}, \ldots, \boldsymbol{\xi}_{M} \in \mathbb{R}$ are the equidistant sampling locations with spacing $\Delta_{x}$, and midpoint $x_{0}=0.5\left(\xi_{1}+\xi_{M}\right)$. We assume that the $m_{i}$ 's are independent, zero-mean, proper complex Gaussian random variables. We further assume that the $m_{i}$ 's are statistically independent of $f(x)$. By putting $s_{i}$ in vector form, we obtain $\mathbf{s}=\left[s_{1}, \ldots, s_{M}\right]^{\mathrm{T}}$.

The cost associated with the $i$ th sample is given by $C_{s_{i}}=\log _{2}\left(\sigma_{s_{i}} / \sigma_{m_{i}}\right)$ and is measured in bits. Here $\sigma_{s_{i}}^{2}=$ $E\left[\left|s_{i}\right|^{2}\right], \sigma_{m_{i}}^{2}=E\left[\left|m_{i}\right|^{2}\right]$, and $\sigma_{s_{i}} / \sigma_{m_{i}}$ is essentially the ratio of the spread of the signal to the spread of the uncertainty, which corresponds to the number of distinguishable levels (dynamic range). Hence the logarithm of this number provides a measure of the number of bits needed to represent this variable. For a field value at a given location, smaller noise levels (smaller $\sigma_{m_{i}}^{2}$ ) correspond to a sample with higher amplitude accuracy and higher cost. On the other hand, a larger noise level corresponds to lower amplitude accuracy and lower cost. Further discussion of this cost function can be found in [11]. Here we will assume that the accuracy and the cost associated with each sample is the same, that is $C_{s_{i}}=C_{s_{1}}, i=1, \ldots, M$. The total cost of the representation is $C_{\mathrm{T}}=\sum_{i=1}^{M} C_{s_{i}}=M C_{s_{1}}$.

The vector $\mathbf{s}$ provides a representation of the random field $f(x)$ in the sense that it is a finite-accuracy finitesampled version of $f(x)$. How accurately does s represent $f(x)$ ? To make this question precise, we can find $\hat{f}(x \mid \mathbf{s})$, the minimum mean-square error (MMSE) estimate of $f(x)$ given $\mathbf{s}$, and examine the error of this estimate, which will, of course, depend on the given bit budget $C_{\mathrm{B}}$. For a given $C_{\mathrm{B}}$, our objective is to choose the number of the samples $M$ and the location of the samples $\xi_{1}, \ldots, \xi_{M}$, while satisfying $C_{\mathrm{T}} \leq C_{\mathrm{B}}$, with the objective of minimizing the mean-square error between $f(x)$ and $\hat{f}(x \mid \mathbf{s})$. This problem can be stated as one of minimizing over $\Delta_{x}, x_{0}$, and $M$ to determine the error

$$
\varepsilon\left(C_{\mathrm{B}}\right)=\min _{\Delta_{x}, x_{0}, M} E\left[\int_{D}\|f(x)-\hat{f}(x \mid \mathbf{s})\|^{2} \mathrm{~d} x\right],
$$

subject to $C_{\mathrm{T}} \leq C_{\mathrm{B}}$. We note that the error is defined between the continuous field $f(x)$ and the estimate $\hat{f}(x \mid \mathbf{s})$. We consider the signal and its estimator in the bounded region $D=\left[x_{L}, x_{H}\right],-\infty<x_{L} \leq x_{H}<\infty$, which may be taken as large as desired. 
The MMSE estimator in $\underline{2}$ can be written as $\hat{f}(x \mid \mathbf{s})=$ $\sum_{j=1}^{M} h_{j}(x) s_{j}=\mathbf{h}(x) \mathbf{s}, \quad$ where $\quad \mathbf{h}(x)=\left[h_{1}(x), \ldots, h_{M}(x)\right]$ [12, Chap. 6]. We note that, given a set of samples, the set of functions $\mathbf{h}(x)$ are the optimal functions that minimize the mean-square error between the actual field and the reconstructed field. Here $\mathbf{h}(x)$ satisfies the equation $\mathbf{K}_{f s}(x)=\mathbf{h}(x) \mathbf{K}_{\mathrm{s}}$ where $\mathbf{K}_{f \mathbf{s}}(x)=E\left[f(x) \mathbf{s}^{\dagger}\right]=$ $\left[E\left[f(x) s_{1}^{*}\right], \ldots, E\left[f(x) s_{M}^{*}\right]\right]$ is the cross covariance between the input field $f(x)$ and the representation vector $\mathbf{s}$, and $\mathbf{K}_{\mathbf{s}}=E\left[\mathbf{s s}^{\dagger}\right]$ is the autocovariance of $\mathbf{s}$. The symbols $*$ and $\dagger$ denote complex conjugate and conjugate transpose, respectively. To determine the optimal linear estimator, one solves this last equation for $\mathbf{h}(x)$. The resulting estimate $\sum_{j=1}^{M} h_{j}(x) s_{j}$ can be interpreted as the orthogonal projection of the unknown random field $f(x)$ onto the subspace generated by the samples $s_{j}$, with $h_{j}(x)$ being the projection coefficients.

Signal model: In our experiments, we use the GaussianSchell model (GSM), a random optical field model with various applications $[13,14]$. A GSM source is characterized by the covariance function

$$
K_{f}\left(x_{1}, x_{2}\right)=A_{f} \exp \left(-\frac{x_{1}^{2}+x_{2}^{2}}{4 \sigma_{I}^{2}}\right) \exp \left(-\frac{\left(x_{1}-x_{2}\right)^{2}}{2 \sigma_{\nu}^{2}}\right) .
$$

Here $A_{f}>0$ is an amplitude coefficient and $\sigma_{I}>0$ and $\sigma_{\nu}>0$ determine the width of the intensity profile and the width of the complex degree of spatial coherence, respectively. For a GSM source, $\beta=\sigma_{\nu} / \sigma_{I}$ may be considered as a measure of degree of global coherence of the field $[\underline{13}, \underline{15}]$. As $\beta$ increases/decreases, the field becomes more coherent/incoherent.

Experiments: In our experiments, we work with the parameters $\sigma_{I}$ and $\beta$. To obtain covariance functions corresponding to random fields with varying degrees of coherence, we use different $\beta$ values: $\beta=1 / 16,1 / 4,1$. For simplicity in presentation, in our simulations we focus on optimizing $\Delta_{x}$ and $M$ and set the less interesting $x_{0}=0$. To compute the error expressions and optimize over the parameters of the representation strategy, we discretize the $x$ space with the spacing $\Delta_{c}$. We approximate the integral in Eq. (2) as $\sum_{k \in D_{N}}\left\|f\left(k \Delta_{c}\right)-\hat{f}\left(k \Delta_{c} \mid \mathbf{s}\right)\right\|^{2} \Delta_{c}$, where $D_{N}=\left\{k: k \Delta_{c} \in D\right\}$. The estimators are only calculated at these discrete points: $\hat{f}\left(k \Delta_{c} \mid \mathbf{s}\right)=\mathbf{h}\left(k \Delta_{c}\right) \mathbf{s}$. To solve the linear equations determining the optimal estimator functions $\mathbf{h}\left(k \Delta_{c}\right)$, we solve the equation $\mathbf{K}_{f s}\left(k \Delta_{c}\right)=\mathbf{h}\left(k \Delta_{c}\right) \mathbf{K}_{\mathbf{s}}$ for each $k \in D_{N}$. For finding the optimum sampling intervals, we use a brute force method, where for a given $C_{\mathrm{B}}$ we calculate the error for varying $\Delta_{x}$ and $M$, and choose the ones providing the best error value. We note that the optimization variable $\Delta_{x}$ and the discretization variable $\Delta_{c}$ are not the same. $\Delta_{c}$ is kept constant throughout all the experiments. We report the error as a percentage defined as $100 \varepsilon\left(C_{\mathrm{B}}\right) / \varepsilon_{0}$, where $\varepsilon_{0}=\int_{-\infty}^{\infty} K_{f}(x, x) \mathrm{d} x=A_{f} \sqrt{2 \pi}$. We choose $x_{H}=-x_{L}=5 \sigma_{I}$.

Trade-offs between the error and the total bit budget: Figure 1 presents the error versus bit budget curves for varying $\beta$. As expected, the error decreases with

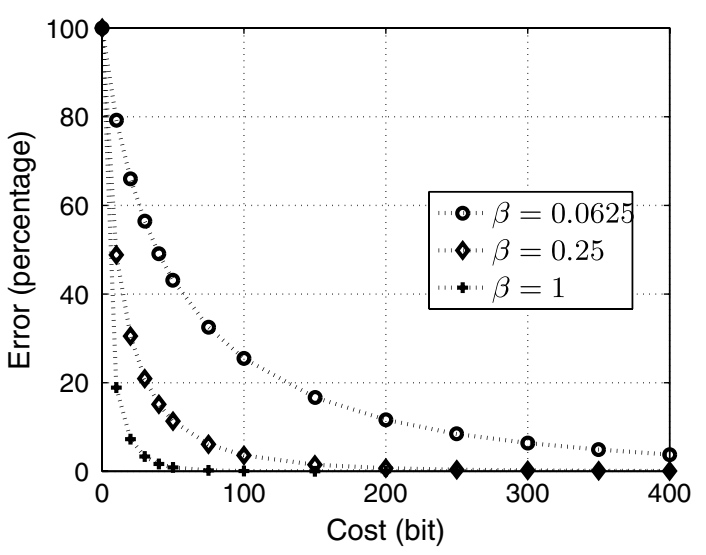

Fig. 1. Error versus cost budget $C_{\mathrm{B}}$ (varying $\beta$ ).

increasing cost budget in all cases. We note that $\varepsilon\left(C_{\mathrm{B}}\right)$ is very sensitive to increases in $C_{\mathrm{B}}$ for smaller $C_{\mathrm{B}}$. Then it becomes less responsive and eventually saturates. We observe that for the relatively incoherent fields (low $\beta$ ), it is more difficult to achieve low values of error within a total bit budget. But as the field becomes more coherent ( $\beta$ increases), the field values at different locations become more correlated with each other, the total uncertainty in the field decreases, and it becomes a lot easier to achieve lower values of error.

We observe that for all values of $\beta$, no matter how small the error tolerance $\varepsilon>0$ is, the continuous finite-energy field can be represented with a finite number of bits. This observation is not surprising since (i) the number of modes needed to effectively represent this source is finite, and (ii) for a given positive error level, the random variable associated with each mode can be represented with a sufficiently large but finite number of bits.

Optimal sampling strategies: We now investigate the relationship between the optimum sampling strategies and the problem parameters $C_{\mathrm{B}}$ and $\beta$. We note that in general the optimum sampling strategies can be interpreted in the light of the competition between the following driving forces: (i) to use as many effectively uncorrelated samples as possible, (ii) to use samples with variances that are as high as possible, and (iii) to have samples that are as highly accurate as possible.

The optimum sampling interval $\Delta_{x}$ and the optimum number of samples $M$ that achieve the errors given in Fig. $\underline{1}$ are presented in Figs. $\underline{2}$ and $\underline{3}$ for $\beta=1 / 16$ and $\beta=1$. (The values of $C_{\mathrm{B}}$ in the figures are $C_{1}=10, C_{2}=20, C_{3}=30, C_{4}=40, C_{5}=50, C_{6}=75$, $C_{7}=100, C_{8}=150, C_{9}=200, C_{10}=250, C_{11}=300$, $C_{12}=350$, and $C_{13}=400$ bits. The observed steps reflect the finite increments used in scanning the optimization space. In Fig. 3 , the optimal points for $C_{4}$ and $C_{5}$ are identical for the increments used.) We observe that in both figures, in general, as $C_{\mathrm{B}}$ increases, the optimum sampling interval decreases and the number of samples increases: when we have more bits to spend, we use a higher number of more closely spaced samples. When $C_{\mathrm{B}}$ is low, the optimal strategy is to use a low number of more distantly spaced samples so that each sample has a reasonable accuracy and each of them provides effectively new information about the field. As allowed cost increases, we can afford more samples with high 


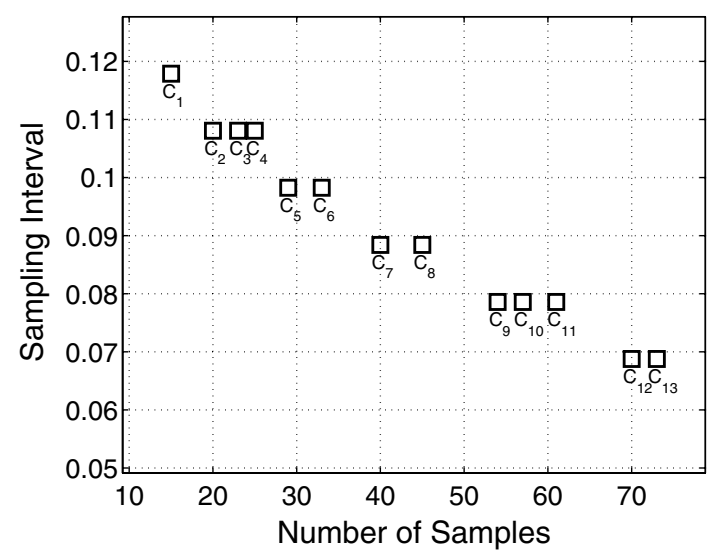

Fig. 2. Optimum sampling interval versus number of samples for different cost budgets $C_{\mathrm{B}}=C_{i}, \beta=1 / 16$.

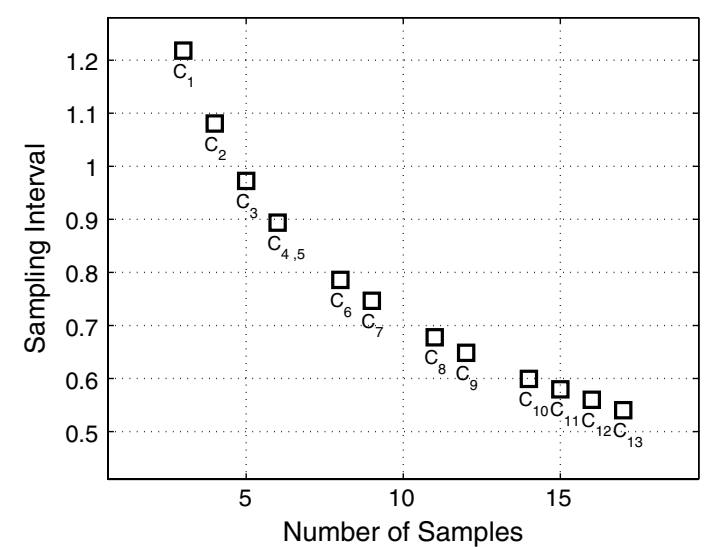

Fig. 3. Optimum sampling interval versus number of samples for different cost budgets $C_{\mathrm{B}}=C_{i}, \beta=1$.

enough accuracies, and we prefer to use more closely spaced samples so that we can get more information about field values we previously had to neglect when the allowed cost was lower.

Note that in Figs. 2 and 3, the cost (hence the accuracy) of a single sample can be found by dividing the cost value to the number of samples. As the allowed cost budget increases, the optimum number of samples increases. However, this increase in the number of samples is, in general, not at the expense of the accuracy of the samples: with increasing budget, the accuracy of the samples also increases.

Comparing Figs. $\underline{2}$ and $\underline{3}$, we observe that when the field is relatively incoherent (Fig. 2), the number of samples used to represent the field is higher, and the sampling intervals are smaller. Under an incoherent GSM source structure, each sample provides information only about field values within its very close neighborhood, and the total uncertainty of the field is spread among many effectively uncorrelated samples. This encourages us to split the budget over a larger number of samples instead of using a smaller number of samples with greater accuracy. It makes sense to choose the spacing of the samples smaller than in a coherent case, since (i) even close samples are uncorrelated and do provide effectively new information, and (ii) the intensity of the field quickly decreases as $|x|$ increases. When the field is more coherent, the field values at different locations are more correlated. In this case, knowing the field at fewer locations with higher accuracy becomes a better strategy. The samples are chosen farther apart compared to the incoherent case to guarantee that each sample provides effectively new information.

In the introduction of this letter, we had posed the question of whether it is better to use a high number of samples with low accuracy or a low number of samples with high accuracy. Our results have provided quantitative answers to this question. The answer strongly depends on the degree of coherence of the field to be represented. When the field is relatively incoherent, it is better to use a larger number of samples with relatively low accuracies. As the field becomes more coherent, using a lower number of samples with higher accuracies is preferred.

A. Özçelikkale was supported by the TÜBITAK Doctoral Scholarship. H. M. Ozaktas acknowledges partial support of the Turkish Academy of Sciences.

\section{References}

1. G. Toraldo Di Francia, J. Opt. Soc. Am. 59, 799 (1969).

2. F. Gori and G. Guattari, J. Opt. Soc. Am. 61, 36 (1971).

3. M. J. Bastiaans, J. Opt. Soc. Am. A 3, 1243 (1986).

4. A. Lohmann, R. Dorsch, D. Mendlovic, Z. Zalevsky, and C. Ferreira, J. Opt. Soc. Am. A 13, 470 (1996).

5. F. T. Yu, Entropy and Information Optics (Marcel Dekker, 2000).

6. A. Burvall, P. Martinsson, and A. T. Friberg, Opt. Lett. 32, 611 (2007).

7. J. J. Healy, B. M. Hennelly, and J. T. Sheridan, Opt. Lett. 33, 2599 (2008).

8. E. D. Micheli and G. A. Viano, J. Opt. Soc. Am. A 26, 1393 (2009).

9. A. Kumar, S. Prabhakar, P. Vaity, and R. P. Singh, Opt. Lett. 36, 1161 (2011).

10. H. M. Ozaktas, S. O. Arik, and T. Coşkun, Opt. Lett. 37, 103 (2012).

11. A. Özçelikkale, H. M. Ozaktas, and E. Arıkan, IEEE Trans. Signal Process. 58, 3607 (2010).

12. H. L. Van Trees, Detection, Estimation and Modulation Theory, Part I (Wiley, 2001).

13. A. Starikov and E. Wolf, J. Opt. Soc. Am. A 72, 923 (1982).

14. A. T. Friberg and J. Turunen, J. Opt. Soc. Am. A 5, 713 (1988).

15. L. Mandel and E. Wolf, Optical Coherence and Quantum Optics (Cambridge Univ., 1995). 